\title{
The chromosome 6q22.33 region is associated with age at diagnosis of type 1 diabetes and disease risk in those diagnosed under 5 years of age
}

\author{
Jamie R. J. Inshaw ${ }^{1,2}$ (D) Neil M. Walker ${ }^{3}$ - Chris Wallace C Leonardo Bottolo $^{4,6,7}$. \\ John A. Todd ${ }^{1,2}$
}

Received: 21 March 2017 / Accepted: 28 July 2017 / Published online: 5 October 2017

(C) The Author(s) 2017. This article is an open access publication

\begin{abstract}
Aims/hypothesis The genetic risk of type 1 diabetes has been extensively studied. However, the genetic determinants of age at diagnosis $(\mathrm{AAD})$ of type 1 diabetes remain relatively unexplained. Identification of AAD genes and pathways could provide insight into the earliest events in the disease process.

Methods Using ImmunoChip data from 15,696 cases, we aimed to identify regions in the genome associated with AAD.

Results Two regions were convincingly associated with AAD $\left(p<5 \times 10^{-8}\right)$ : the MHC on 6p21, and 6q22.33. Fine-mapping of $6 \mathrm{q} 22.33$ identified two AAD-associated haplotypes in the region nearest to the genes encoding protein tyrosine phosphatase receptor kappa (PTPRK) and thymocyte-expressed molecule involved in selection (THEMIS). We examined the susceptibility to type 1 diabetes at these SNPs by performing a metaanalysis including 19,510 control participants. Although these SNPs were not associated with type 1 diabetes overall $(p>0.001)$, the SNP most associated with AAD, rs72975913, was associated with susceptibility to type 1 diabe-
\end{abstract}

Electronic supplementary material The online version of this article (https://doi.org/10.1007/s00125-017-4440-y) contains peer-reviewed but unedited supplementary material, which is available to authorised users.

Jamie R. J. Inshaw

jinshaw@well.ox.ac.uk

John A. Todd

jatodd@well.ox.ac.uk

1 JDRF/Wellcome Trust Diabetes and Inflammation Laboratory, Wellcome Trust Centre for Human Genetics, University of Oxford, NIHR Oxford Biomedical Research Centre, Nuffield Department of Medicine, Roosevelt Drive, Oxford OX3 7BN, UK

2 JDRF/Wellcome Trust Diabetes and Inflammation Laboratory, Cambridge Institute for Medical Research, University of Cambridge, Cambridge, UK tes in those individuals diagnosed at less than 5 years old $\left(p=2.3 \times 10^{-9}\right)$.

Conclusion/interpretation PTPRK and its neighbour THEMIS are required for early development of the thymus, which we can assume influences the initiation of autoimmunity. Non-HLA genes may only be detectable as risk factors for the disease in individuals diagnosed under the age 5 years because, after that period of immune development, their role in disease susceptibility has become redundant.

Keywords Age at diagnosis · Early diagnosis - Genetic risk · Type 1 diabetes

$\begin{array}{ll}\text { Abbreviations } \\ \text { AAD } & \text { Age at diagnosis } \\ \text { ASP } & \text { Affected sib-pair } \\ \text { FDR } & \text { False discovery rate }\end{array}$

3 Clinical Informatics, Cambridge University Hospitals NHS Foundation Trust, Cambridge, UK

4 Department of Medicine, University of Cambridge, Cambridge, UK

5 MRC Biostatistics Unit, Cambridge Institute of Public Health, Cambridge, UK

6 Department of Medical Genetics, University of Cambridge, Cambridge, UK

7 The Alan Turing Institute, London, UK 


$\begin{array}{ll}\text { gMPPI } & \begin{array}{l}\text { Group marginal posterior probability of } \\ \text { inclusion }\end{array} \\ \text { GRID } & \begin{array}{l}\text { Genetic Resource Investigating Diabetes } \\ \text { Genome-wide association study } \\ \text { GWAS }\end{array} \\ \text { IDDMGEN } & \begin{array}{l}\text { Tyypin 1 Diabetekseen Sairastuneita } \\ \text { Perheenjäsenineen }\end{array} \\ \text { LD } & \text { Linkage disequilibrium } \\ \text { NI } & \text { Northern Ireland } \\ \text { T1DGC } & \text { Type 1 Diabetes Genetics Consortium } \\ \text { T1DGEN } & \text { Tyypin 1 Diabeteksen Genetiikka }\end{array}$

\section{Introduction}

Since the introduction of genome-wide association studies (GWAS), over 50 regions in the genome have been associated with susceptibility to type 1 diabetes [1-6], but less research has examined the genetic determinants of age at diagnosis (AAD) of type 1 diabetes. One study, limited to specific SNPs in regions associated with type 1 diabetes, identified that the MHC, IL-2 (IL2) and renalase $(R N L S)$ gene regions showed evidence of association with AAD [7, 8]. However, the question has never been examined in a genome-wide fashion. Identification of genes associated with the initiation of the anti-islet autoimmunity, which is in most cases established by the age of 3 years [9], could help to establish the earliest events in the disease process.

Here we aimed to identify genetic determinants of AAD in a more powerful approach using data from an extensive SNP panel, the custom array ImmunoChip [4], by combining data from independent cases and affected sib-pairs (ASPs) to increase sample size and improve the genetic map through imputation.

\section{Methods}

We analysed data from six cohorts, independent cases from the UK Genetic Resource Investigating Diabetes (GRID) cohort [2], the Northern Irish GRID (NI) cohort (used here for the first time), and the Finnish IDDMGEN (Tyypin 1 Diabetekseen Sairastuneita Perheenjäsenineen) and T1DGEN (Tyypin 1 Diabeteksen Genetiikka) cohorts [10], in addition to ASPs from the Type 1 Diabetes Genetics Consortium (T1DGC) cohort [11] (from north America, Europe, Asia and the UK) and the UK Warren cohort [12]. The majority of individuals were diagnosed in childhood, with $92 \%$ diagnosed at less than 20 years of age. Genotyping (see electronic supplementary material [ESM] Genotyping) was performed on 16,015 affected individuals, $8683(54 \%)$ independent participants and 7332 (46\%) ASPs (Table 1). Quality control was performed prior to analysis to minimise the risk of reporting false-positive results (ESM Quality control, ESM Figs 1-3).
SNP imputation Where mentioned, we used IMPUTE2 software (http://mathgen.stats.ox.ac.uk/impute/impute_v2.html) [13, 14], using 1000 Genomes Project data [15] (version III) as the reference dataset (https://mathgen.stats.ox.ac.uk/ impute/1000GP Phase3.html) to perform SNP imputation. We excluded SNPs with a minor allele frequency of less than 0.01 or an imputation information score less than 0.8 as a quality control measure (an imputation score of 0 indicates no certainty in the imputed genotype, whereas a score of 1 indicates no uncertainty in the imputed genotype).

Association discovery using ImmunoChip data As we had a population that comprised related and independent cases, from a variety of cohorts containing individuals from multiple countries between and within cohorts, it was crucial to account for population structure, to avoid reporting spurious associations. We did this by performing an inverse-variance weighted meta-analysis [16], in which we stratified the samples by cohort and examined the effect of each SNP on $\log _{e}$ AAD (assuming an additive mode of inheritance), adjusting for sex and the top five principal components within the cohort to account for population structure.

However, in cohorts with related individuals, standard principal components analysis may not correctly identify the population structure, as the population-level clusters are confounded by the relatedness between individuals. Therefore, in these cohorts, we used principal components analysis in related-samples, PC-AiR [17], which estimates principal components by identifying a subset of genetically dissimilar individuals and performs principal components analysis on this subset, before using these principal components to estimate the ancestry of the remaining individuals in the cohort. This was performed using the GENESIS R package (https:// www.bioconductor.org/packages/devel/bioc/html/GENESIS. $\mathrm{html}$, version 2.2.7) [18]. We applied a variance-components model using the GenABEL R package (http://www.genabel. org/packages/GenABEL, version 1.8-0, Grammar-Gamma method) [19] to analyse the effect of each SNP in cohorts of related individuals, which takes into account relatedness between individuals. ESM Fig. 4 provides a schematic overview of the association discovery meta-analysis procedure.

We also performed the association discovery analysis using an alternative approach. First, we fitted a linear mixed model [20] with the $\log _{\mathrm{e}} \mathrm{AAD}$ as the outcome, adjusting for sex as a fixed effect and including random effects for cohort, country and family identifier. We then used the residuals from the linear mixed model as the outcome variable and tested the association of each SNP using a linear regression model. We called this approach the 'residual-based model', and it has been proposed by Aulchenko et al [21] to make genomewide analysis for related individuals possible. Since our aim was eventually to fine-map statistically significant regions, the advantage of the residual-based model is that the residuals can 
Table 1 Baseline characteristics and inclusion in the primary AAD analysis after quality control

\begin{tabular}{lllcccc}
\hline Cohort & Country & Type & Genotyped & Included & AAD: median (IQR) & SNPs after QC \\
\hline GRID & UK & Independent & 6799 & 6736 & $8(4,11)$ & 164,953 \\
IDDMGEN & Finland & Independent & 1111 & 1073 & $9(5,12)$ & 156,343 \\
NI & NI & Independent & 524 & 509 & $7(4,10)$ & 156,343 \\
T1DGEN & Finland & Independent & 249 & 249 & $16(10,24)$ & 156,343 \\
Warren & UK & ASP & 907 & 839 & $10(5,15)$ & 156,343 \\
T1DGC & Asia & ASP & 960 & 919 & $10(5,14)$ & 167,537 \\
T1DGC & Europe & ASP & 2521 & 2485 & $11(6,17)$ & 167,537 \\
T1DGC & USA & ASP & 2593 & 2544 & $8(4,13)$ & 167,537 \\
T1DGC & UK & ASP & 351 & 342 & $8(4,11)$ & 167,537 \\
Total & All & All & 16,015 & 15,696 & $9(5,12)$ & 150,381 \\
\hline
\end{tabular}

The intersect of the SNPs that passed QC across genotype batch were included in the analysis. Total SNPs after QC refers to the common set of SNPs across all collections

QC, quality control also be used as the outcome variable in a fine-mapping analysis for continuous traits. ESM Fig. 5 illustrates the steps of this second approach used for variants discovery.

SNPs were declared to be associated with AAD if the $p$ value was less than a genome-wide significance threshold of $5 \times 10^{-8}$. We also highlight regions associated with a false discovery rate (FDR) [22] of less than 0.05 , to identify the regions next most likely to influence AAD. In this analysis, we used a stringent definition, removing the MHC region before calculating the FDR, as including all the highly associated SNPs from the MHC can inflate the threshold below which SNPs are declared to be associated, increasing the probability of reporting false-positive results.

To add further evidence to the detected associated SNPs, we combined cases with 19,510 control individuals (ESM Table 1) and performed an inverse-variance weighted meta-analysis across cohorts, examining the effect of the SNPs on risk of type 1 diabetes overall and in those who were diagnosed at less than 5 years of age. Cohorts of independent individuals were analysed by fitting a logistic regression model adjusting for the top five principal components and examining the effect of the SNP of interest on risk of type 1 diabetes. Cohorts of related individuals were analysed using a generalised linear mixed model association test [23], using the GMMAT R package (https://www.hsph.harvard.edu/han-chen/software/, version 0 . 7-1), adjusting for the top five principal components as fixed effects, and using a kinship matrix to define the covariance structure of the random effect included in the model. We present ORs for the SNPs associated with AAD for their association with type 1 diabetes overall and for those diagnosed at less than 5 years of age, to compare the direction of effect between analyses, with a consistent direction of effect adding further evidence that the association was genuine.

Association discovery: imputed data A subset of 1768 cases from the GRID cohort had data that had been genotyped using the Affymetrix GeneChip Mapping 500K, and 3833 had been genotyped using the Illumina $550 \mathrm{~K}$ Infinium microarray platform [3], both of which are GWAS chips and cover a broader spectrum across the genome than the ImmunoChip. We stratified the GRID cohort into strata, one containing cases who had been genotyped using Affymetrix technology, and the other containing cases who had been genotyped using Illumina. We imputed in $1 \mathrm{Mb}$ blocks across the entire genome, and then tested the association of each SNP with $\log _{\mathrm{e}}$ AAD for both strata using SNPTEST software (version II, using the frequentist option, https://mathgen.stats.ox.ac.uk/genetics software/snptest/snptest.html) [24], before combining results by an inverse-variance weighted, fixed-effects meta-analysis. Owing to an uncertain genotype call, we used the expectation maximisation option to estimate the association of each SNP while performing the imputation of the missing genotypes.

Fine-mapping In AAD-associated regions $\left(p<5 \times 10^{-8}\right)$, we imputed SNPs to obtain the densest SNP set possible in that region, and performed fine-mapping to identify groups of candidate causal SNPs. We used GUESSFM (https://github.com/ chr1swallace/GUESSFM version 1.0.1), [25] a fine-mapping algorithm $[26,27]$ that allows more than one causal SNP in the region to be identified. Briefly, GUESSFM identifies models, that is, combinations of SNPs, with high posterior support given the likelihood and the priors, by carrying out a stochastic search. Models are ranked by the frequency with which they appear in the search. The SNPs included in the most visited models are most likely to be causally associated with AAD. In contrast to other fine-mapping methods that use summary statistics, GUESSFM makes use of raw genotype data. One output from GUESSFM is the group marginal posterior probability of inclusion (gMPPI), which groups SNPs in tight linkage disequilibrium (LD) and can be thought of as the posterior support that exactly one of the SNPs in the group is causal. 
The outcome variable was the same set of residuals used in the association discovery analysis residual-based model, so population and family structure had been accounted for. Our primary analyses used the default prior for the expected number of causal variants in the region to be three. For reproducibility of the results, we examined also the effect of changing the expected number of causal SNPs to two or six. For comparison, we fitted stepwise linear regression models in each region of interest (with the same set of residuals as the outcome), initially including the most significant SNP in the region, followed by the most associated SNP conditional on the initial SNP. We repeated this process until one of the SNPs in the model fell below a commonly used [25, 28] significance threshold of $p=5 \times 10^{-6}$.

Haplotype and diplotype analyses The output from GUESSFM identifies groups of candidate causal SNPs in LD that are most likely to be associated with the phenotype. We examined these groups detected by GUESSFM in a haplotype analysis to highlight transmission patterns and to visualise haplotype membership of the selected SNPs. We phased SNPs using SNPHAP software (https://github.com/chr1swallace/ snphap, version 1.3), which generates the posterior probability of each haplotype for each individual. To capture the uncertainty of the haplotype phase, we simulated ten haplotype datasets, where, in each dataset, the haplotype for every individual was sampled from their haplotype posterior distribution. The effect size of each haplotype relative to the most common was calculated in each dataset using a linear mixed model, with $\log _{\mathrm{e}} \mathrm{AAD}$ as the outcome, adjusting for haplotype and sex as fixed effects, and family identifier, cohort and country as random effects. Results were pooled across the ten datasets using the mice R package (http:/www. jstatsoft.org/v45/i03/, version 2.9) for combining results from multiply imputed datasets [29]. Once haplotypes had been estimated, we extended the analysis to examine diplotypes by combining the haplotypes from the two chromosomes and using the methods described above to estimate the effect of each diplotype.

All analyses were carried out using $\mathrm{R}$ version 3.3.2.

All samples were collected after approval from the relevant research ethics committees, and written informed consent was obtained from the participants.

\section{Results}

Association discovery ImmunoChip analysis Results from the meta-analysis identified two regions that were associated at $p<5 \times 10^{-8}$, the $\mathrm{MHC}$ region and the $6 \mathrm{q} 22.33$ region, the latter of which contains the genes encoding protein tyrosine phosphatase receptor kappa $(P T P R K)$ and thymocyteexpressed molecule involved in selection (THEMIS) (Fig. 1).
The index SNP in the MHC was rs9273363 $\left(p=2.16 \times 10^{-35}\right)$, which has previously been shown to be associated with type 1 diabetes [30], to tag the HLA DQB1*03:02 genotype [31] and to be located in a potential enhancer region of the major type 1 diabetes gene, HLA-DQB1 [32].

In the chromosome $6 \mathrm{q} 22.33$ region, the lead SNP was rs72975913 $\left(p=2.94 \times 10^{-10}\right)$, which is in $\operatorname{LD}\left(r^{2}=0.99\right)$ with a key SNP associated with coeliac disease (rs72975916) [33]. Results from the residual-based model were similar, with the same two regions, and no others, reaching genome-wide significance. Manhattan and quantile-quantile plots for the residual-based model can be found in ESM Fig. 6, and residual plots for this analysis in ESM Figs 7 and 8.

The results from the residual-based model estimate that the addition of an A allele (the minor allele in controls) at the lead MHC SNP, rs9273363, is associated with an $11.8 \%$ decrease in AAD, which translates to a decrease of 1.11 years (13 months). The addition of the major $\mathrm{C}$ allele at the lead 6q22.33 SNP, rs72975913, is associated with a younger AAD by 0.7 years ( 8 months). If an individual is homozygous for the AAD risk allele at the lead SNP in both regions, they are estimated to be diagnosed 4.12 years younger than those who are homozygous for the non-risk allele at both loci.

Although there were just two regions that reached genomewide significance, Table 2 shows that region 1q24.3, which contains the Fas ligand (FASLG) gene, contains at least one SNP with some evidence (FDR $<0.05$ ) of association with AAD. It adds weight to the evidence that this SNP might be truly related to AAD since the type 1 diabetes risk effect direction in those diagnosed at less than 5 years of age is the same as the AAD effect direction for each SNP, that is, the risk allele for type 1 diabetes is associated with younger AAD.

Association discovery analysis-imputed data In total, 7,476,246 SNPs were examined for their association with AAD. Just one region reached genome-wide significance, the $\mathrm{MHC}$ region (ESM Fig. 9), which indicates that there was not sufficient power to detect associated regions outside the ImmunoChip due to a smaller sample size in this analysis (5601 vs 15,696 in the ImmunoChip analysis).

Fine-mapping It is beyond the scope of the current study to consider fine-mapping of the $\mathrm{MHC}$ region, and it is well established that the HLA-DQB1,HLA-DRB1,HLA-A and $H L A-B$ genes are the primary determinants of the MHC risk in type 1 diabetes [30].

In the 6q22.33 region (positions $127,952,182$ to $128,340,790$ on chromosome 6 , NCBI build 37 , https:// www.ncbi.nlm.nih.gov), we performed SNP imputation with a concordance of $97.9 \%$ (i.e. for each SNP of known genotype, the imputed genotype matched the known genotype at least $97.9 \%$ of the time). In total, 786 SNPs were analysed in this region, including 319 imputed SNPs. 
a

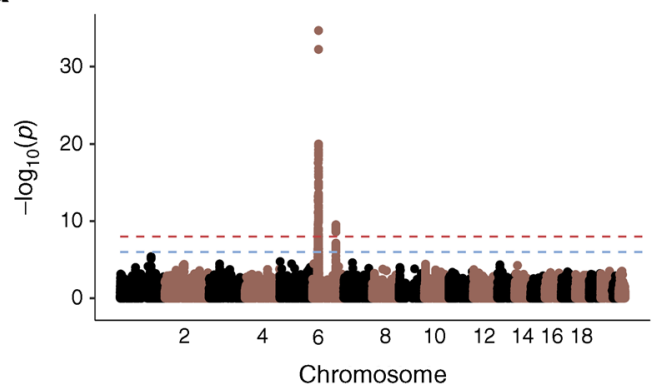

b

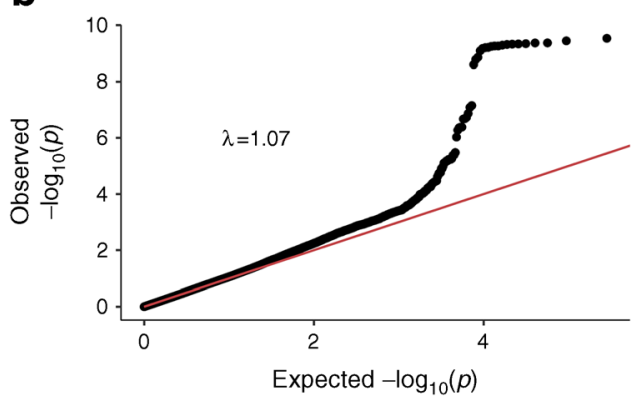

C

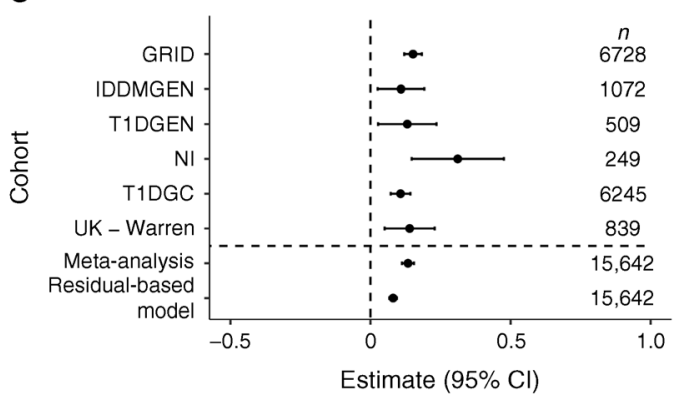

d

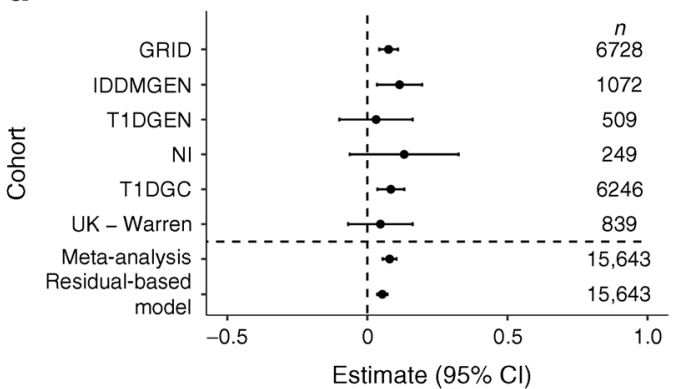

Fig. 1 (a) Manhattan plot from the association discovery meta-analysis for AAD of type 1 diabetes. (b) Quantile-quantile plot (excluding the MHC region). (c) Forest plot for the lead SNP in the MHC region, rs9273363. (d) Forest plot for the lead SNP in the 6q22.33 region, rs72975913

The GUESSFM analysis highlighted three potential signals in the region (Fig. 2, ESM Table 2). Group 1 comprised 22 SNPs, had a gMPPI of 0.50 and was in LD $\left(r^{2}=0.49\right)$ with group 2, which contained 12 SNPs and had a gMPPI of 0.42 ; this group contains rs 802719 , the SNP with the highest posterior support (SNP marginal posterior probability of inclusion $=0.14)$. Finally, group 3 , with the strongest signal, contained 24 SNPs and had a gMPPI of 0.96 , including the index SNP from the association discovery analysis, rs72975913. However, the LD between groups 1 and 2 implies that these groups are probably the same signal; in addition, the fact that the SNP with the highest posterior probability is contained in group 2 rather than group 1 implies that the true signal is more likely to be from group 2 . The results were similar when changing the prior number of SNPs expected in the model to two or six, both showing three signals in the region. Results from the stepwise linear regression approach indicated that there were two signals: the lead SNP once imputed data was included was SNP rs11753289, which is contained in group 3 from the GUESSFM analysis, with a borderline second signal, from SNP rs802719 $\left(p=7.5 \times 10^{-6}\right)$, which is contained in group 2 .

Haplotype and diplotype analyses In the $6 \mathrm{q} 22.33$ region, we examined the 58 SNPs contained in groups 1,2 and 3 from the GUESSFM analysis and estimated the effect of each haplotype relative to the most common, which was the major allele at each of the 58 SNPs. The minor allele haplotype from the group $2 \mathrm{SNPs}$ is associated with younger AAD, while the minor allele haplotype from the group 3 SNPs is associated with an older AAD (Fig. 3). The LD between groups 1 and 2 can be observed, and the two main signals in the region appear to be from groups 2 and 3. Extending the analysis to examine diplotypes showed that being heterozygous at group 2 SNPs leads to an estimated $3.1 \%$ decrease in $\mathrm{AAD}$ relative to the most common diplotype, which translates to an estimated younger AAD of 3.1 months. Having two copies of the minor group 2 SNPs leads to an estimated younger AAD of $8.3 \%$, which translates to 8.1 months. In contrast, individuals with one copy of the minor group 3 haplotype have an estimated AAD 6.4\% (6.8 months) older than those with the most common diplotype, which increases to $11.9 \%$ (12.9 months) if they have two copies of the minor group 3 haplotype (Fig. 3). These results show there are two main risk haplotypes that predispose to younger $\mathrm{AAD}$ in the region: the minor allele haplotype for group 2 and the major allele haplotype for group 3 .

Type 1 diabetes risk analysis We examined one SNP from each of groups 2 and 3 (rs802719 and rs72975913, respectively) from the fine-mapping analysis and examined the effect of these SNPs on type 1 diabetes risk in a meta-analysis including the control participants from ESM Table 1. As expected from the lack of previously reported associations in this region, neither of the SNPs was strongly associated with type 1 diabetes overall $(p>0.001)$ (forest plots are shown in ESM Fig. 10).

However, owing to the association with $\mathrm{AAD}$, we stratified each cohort into age group at diagnosis $(0-4.99,5-9.99$, $10-14.99,15-19.99$ and $\geq 20$ years) and assessed the 


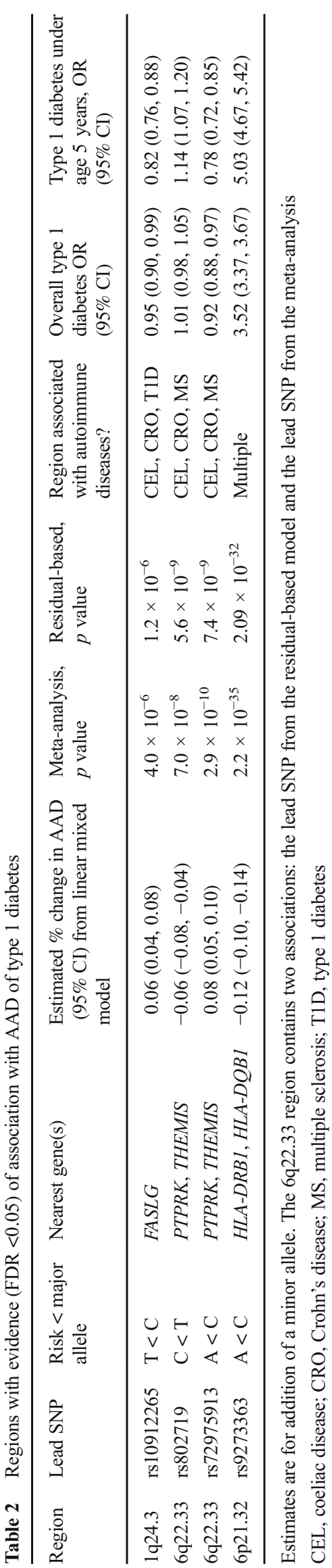


Fig. 2 Output from GUESSFM fine-mapping the $6 \mathrm{q} 22.33$ region. (a) Location of the PTPRK and THEMIS genes, the closest genes to the candidate causal SNPs. (b) Map of the candidate causal SNPs to their physical location along chromosome 6. (c) The dots depict the strength of association (marginal posterior probability of inclusion) for each SNP, while the height of the shaded region is the gMPPI, the probability that one of the SNPs in the group is causal for $\mathrm{AAD}$. It shows three signals in the region, termed groups 1 (blue), 2 (red) and 3 (green). (d) LD between SNPs

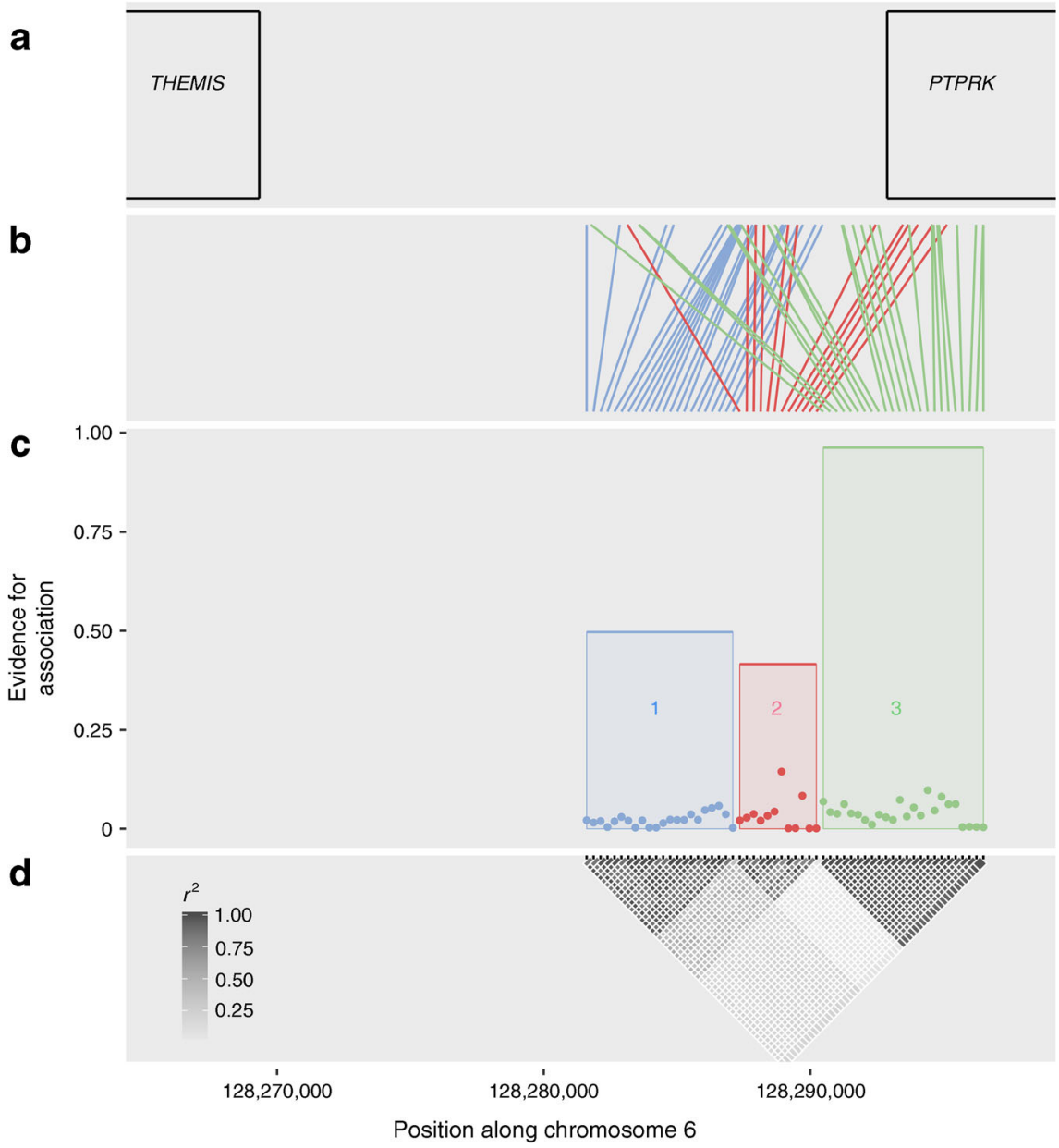

association of both SNPs with risk of type 1 diabetes within strata. A strong association was observed in the youngest AAD category at rs72975913 $\left(p=2.3 \times 10^{-9}\right.$, OR 0.78 , $n=3807$ ) and evidence of an association at rs802719 ( $p=2.2 \times 10^{-5}$, OR $\left.1.14, n=3806\right)$, with the forest plots shown in ESM Fig. 11. The effect estimate was in the same direction as the AAD analysis, with the minor A allele associated with decreased disease susceptibility in those diagnosed at less than 5 years old at rs72975913, which is associated with an older AAD, and for rs802719 the minor C allele increases disease susceptibility and decreases AAD. To examine the sensitivity of our results to the cut-off for which to define the youngest AAD strata, we repeated the analysis, defining the youngest age category first as $<4$ years and then as $<6$ years. The results did not change significantly, with rs72975913 remaining associated on a genome-wide basis regardless of cut-off and with a similar effect size observed at rs802719 (ESM Table 3). There is a trend showing how the effect size on disease susceptibility decreases at both SNPs as the age group at diagnosis increases, indicating that the genetic effects in this region act in those diagnosed at a young age (Fig. 4, ESM Figs 12 and 13 for minor allele frequencies). This may explain why this region has not previously been associated with type 1 diabetes as, typically, GWAS datasets include cases who have been diagnosed across a range of ages.

\section{Discussion}

In the first ImmunoChip analysis examining the AAD of type 1 diabetes, we found, as expected, that the MHC was the major genetic influence, while the $6 \mathrm{q} 22.33$ region was a second associated region. We performed fine-mapping across the 6q22.33 region, which contains the adjacent PTPRK and THEMIS genes, and has never previously been associated with AAD [7, 8] or type 1 diabetes, but has been reported to be associated with susceptibility to other autoimmune diseases [33-35].

We identified two haplotypes in the region that were associated with younger AAD. These haplotypes showed evidence of influencing type 1 diabetes susceptibility in those diagnosed at less than 5 years of age but not for other age groups. This implies that the region impacts on risk of type 1 diabetes at a young age but not once the immune system is more fully developed. In an era of increasing sample sizes, this study highlights the benefit of refining a phenotype in order to 

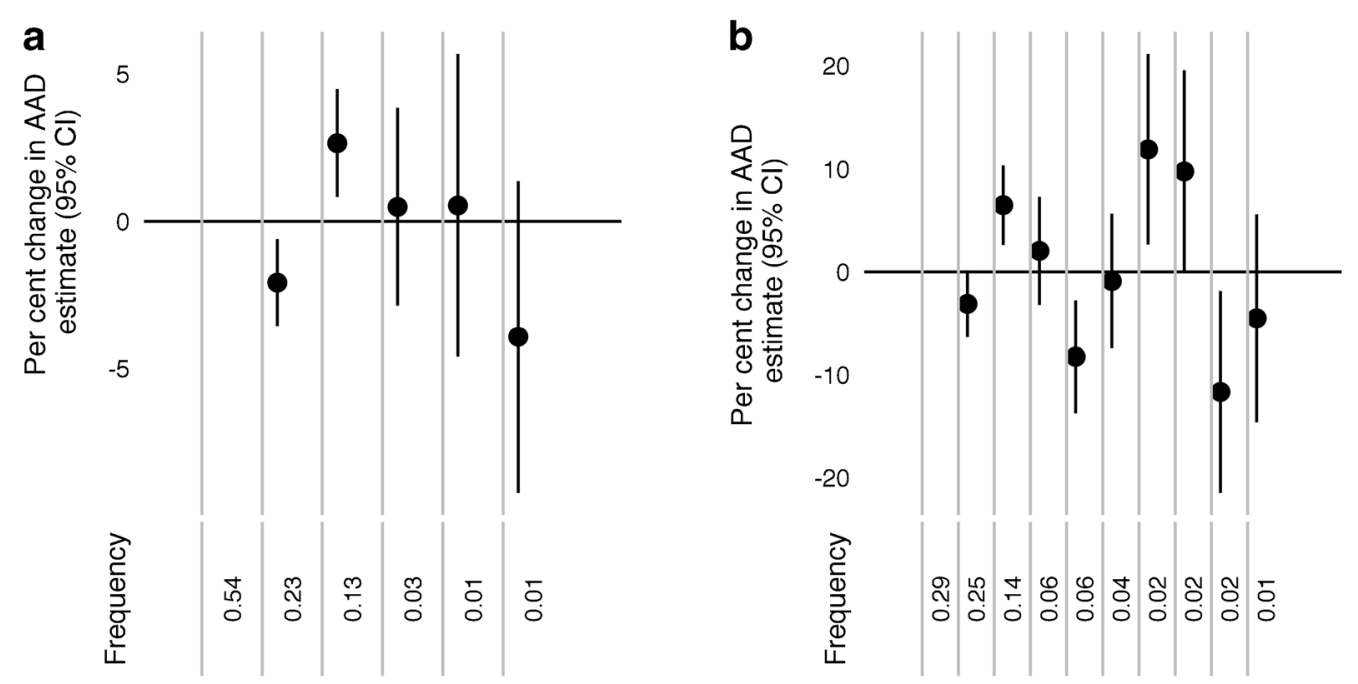
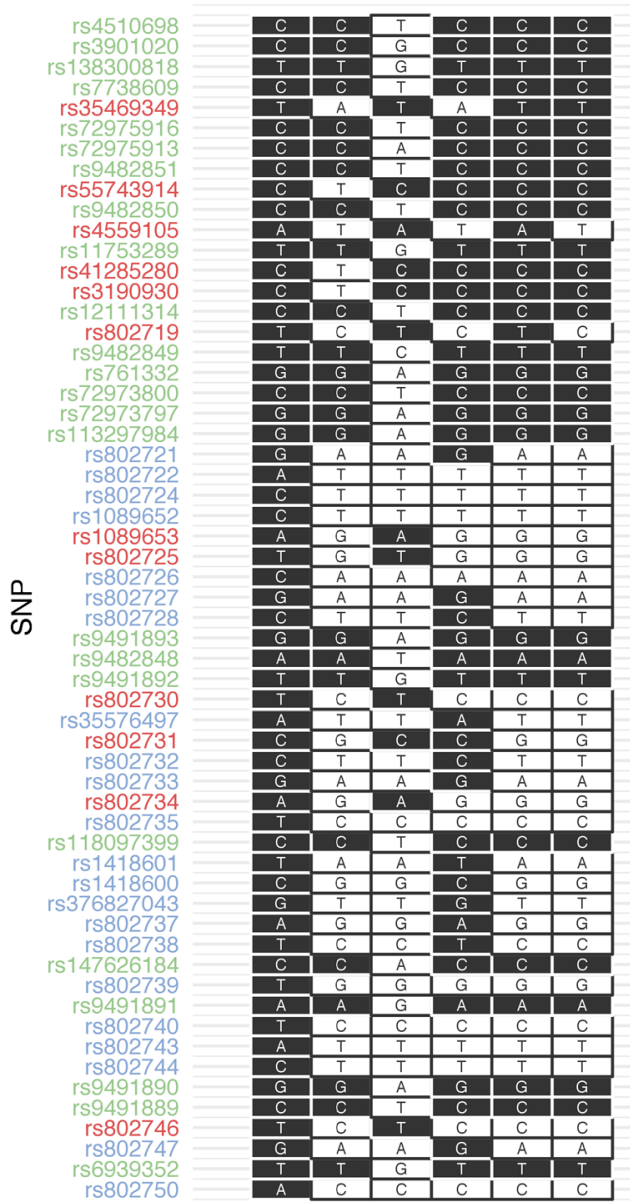

Haplotype

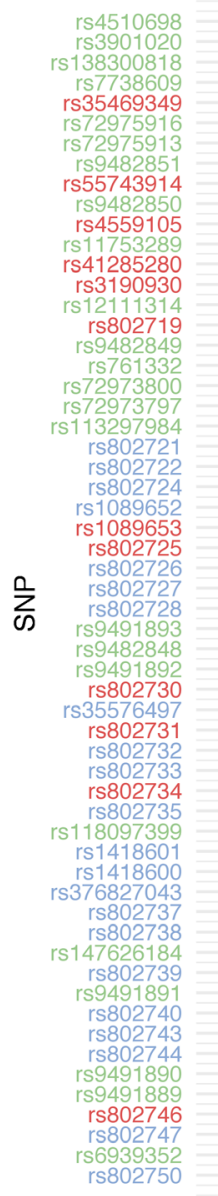

2 (red) and 3 (green). Black, major alleles; white, minor alleles. (b) Diplotype analysis of the same region. Black, major homozygotes; grey, heterozygotes; white, minor homozygotes

identify the main genetic determinants in a particular subset. Analyses of the immunology and pancreas histology of type 1 diabetes do reveal distinct autoimmune features in children diagnosed under age 5 years [36]. Genetic findings such as 
Fig. 4 (a) Risk of type 1 diabetes (T1D) at SNPs contained in group 3 (GUESSFM analysis) rs 72975913 , stratified by AAD group. (b) Risk of type 1 diabetes at the SNPs contained in group 2, rs 802719 , stratified by AAD group. The effect size is for addition of a minor allele at the loci, assuming an additive mode of inheritance on the log-odds scale
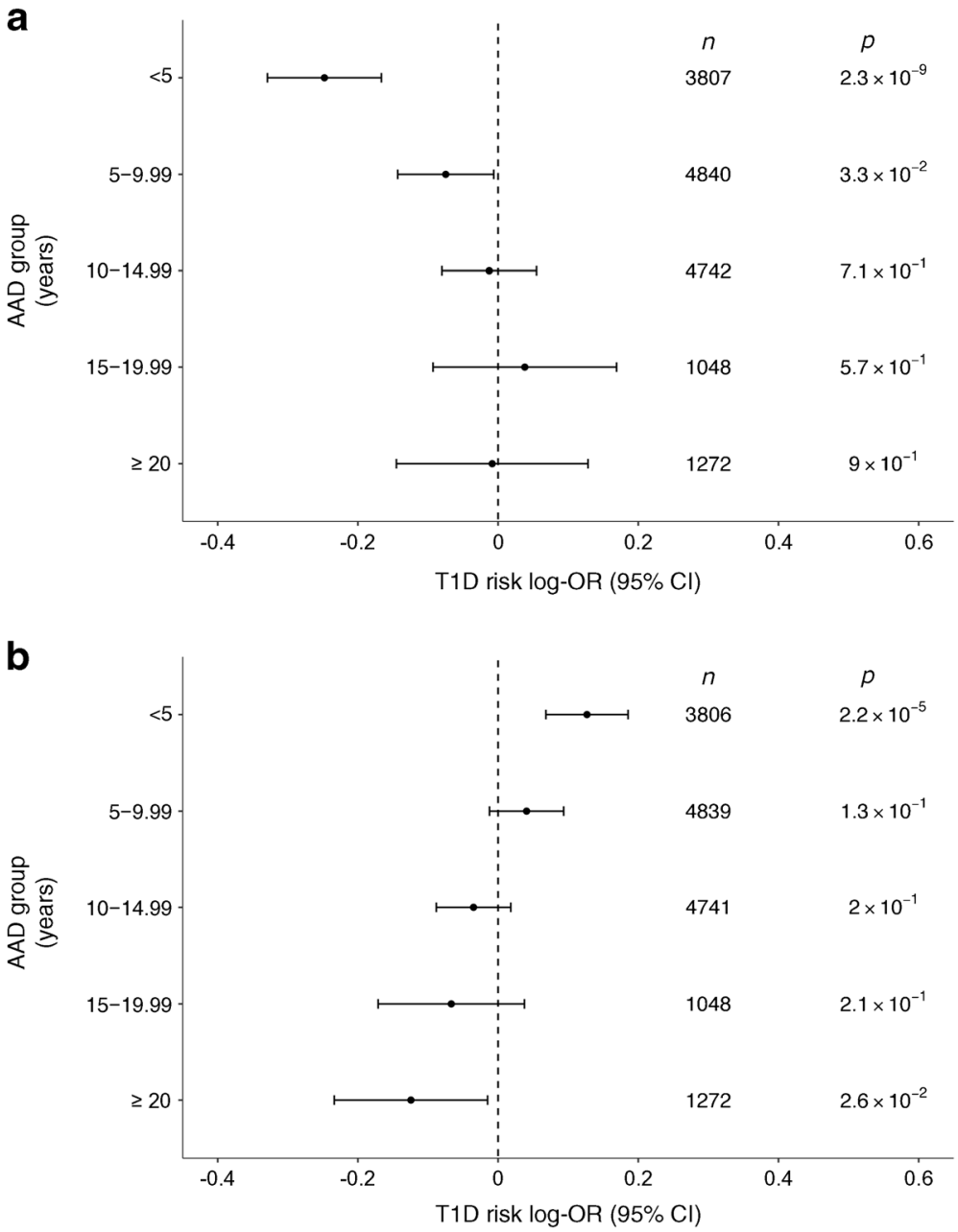

ours will help to identify the key cells and tissues involved, pointing, in this case, to the thymus being particularly important in early, aggressive disease.

PTPRK and THEMIS are both important for transition of double-positive $\left(\mathrm{CD}^{+}, \mathrm{CD}^{+}\right)$thymocytes to single-positive thymocytes [37, 38], and there is a reduction in number of mature $\mathrm{CD}^{+} \mathrm{T}$ cells in mice that have both genes knocked out, over and above the effect that each gene has independently, indicating that they are both vital to thymopoiesis [39]. Chromosome conformation capture analyses have identified the PTPRK promoter as a target of disease-associated sequences [40], supporting its candidacy as the causal gene for AAD.

The 6 q22.33 region has been associated with other autoimmune diseases: the index SNP for coeliac disease is rs55743914 [33], which is contained in group 2 in our analysis, and the minor allele is associated with increased risk of coeliac disease and also younger AAD for type 1 diabetes. The secondary signal for coeliac disease (rs72975916) is contained in group 3 , and the minor allele is associated with reduced risk of coeliac disease and older AAD for type 1 diabetes, so the directions of effect between AAD of type 1 diabetes and coeliac disease are consistent.
However, the lead SNP for multiple sclerosis, rs802734 [35], which is contained in group 2 in this analysis, has the opposite direction of effect (the minor allele being protective against multiple sclerosis). This signal in multiple sclerosis was not replicated in a larger ImmunoChip analysis [41], and hence the risk of multiple sclerosis at this SNP may also depend on another factor, for example age at onset. Just one signal was detected in the region as associated with Crohn's disease, rs9491891 [42], which is contained in group 3, with a direction of effect also opposite to that for type 1 diabetes and coeliac disease. The age at onset of multiple sclerosis and Crohn's disease in the cohorts in these analyses is older than the AAD of the type 1 diabetic individuals in our dataset, while there can be a long delay in coeliac disease between onset and diagnosis [43], so it may be that the difference in effect direction could be to do with a changing immune system with age.

Another possibility is that the SNPs affecting earlydiagnosed type 1 diabetes are affecting a different pathway, tissue or cell type from the same SNPs that have the opposite effect in multiple sclerosis and Crohn's disease; that is, an increased level of a protein in one cell type might increase 
the risk of type 1 diabetes, while the increase in that same protein in a different cell type might protect against multiple sclerosis or Crohn's disease. There is no evidence that the $6 q 22.33$ region is associated with age at onset of multiple sclerosis or Crohn's disease, although there are very few individuals in these analyses who were diagnosed in childhood $[35,42]$ and it is difficult to assess in coeliac disease given the time between onset and diagnosis [43]. We hypothesise that there is co-localisation in the region between AAD of type 1 diabetes and coeliac disease, given the similar genetic risk variants and also the fact that individuals diagnosed with type 1 diabetes at a young age are more likely to have coeliac disease [44]. Our analysis offers a genetic explanation for this phenomenon.

There was some evidence (FDR $<0.05$ ) of an association with AAD at one other region, 1q24.3. This region contains the FASLG gene and has been shown to be associated with type 1 diabetes itself [5]; therefore it might be involved in a pathway that acts early in the disease course of type 1 diabetes, leading to the anti-islet autoimmunity that we now know is established in most cases by the age of 3 years $[9,45]$.

A potential limitation of our study is that the majority $(92 \%)$ of individuals with type 1 diabetes were diagnosed at less than 20 years old, and it is unlikely that we have identified all the variants associated with the AAD of type 1 diabetes. However, there is scope to perform a similar analysis in a population with more individuals diagnosed at over 18 years of age when data are generated in the future. Finally, some caution should be taken when interpreting the association at 1q24.3 (near FASLG), as the association did not reach a stringent genome-wide significance.

In conclusion, we have identified a novel AAD region at $6 \mathrm{q} 22.33$, as well as confirmed the well-established association of the MHC. The two risk haplotypes at 6q22.33 show evidence of association with type 1 diabetes in individuals diagnosed at less than 5 years of age and might thus guide therapeutic strategies in those with early-diagnosed type 1 diabetes.

\footnotetext{
Acknowledgements We gratefully acknowledge all individuals who provided biological samples or data for this study. This research uses resources provided by the T1DGC, a collaborative clinical study sponsored by the National Institute of Diabetes and Digestive and Kidney Diseases (NIDDK), the National Institute of Allergy and Infectious Diseases (NIAID), the National Human Genome Research Institute (NHGRI), the National Institute of Child Health and Human Development (NICHD) and the JDRF, and supported by grant U01 DK062418 from the US National Institutes of Health. Further support was provided by grants from the JDRF (9-2011-253 and 5-SRA-2015130-A-N) and the Wellcome Trust (091157 and 107212) to the Diabetes and Inflammation Laboratory at Oxford and Cambridge Universities.

We obtained DNA samples from the British 1958 Birth Cohort collection, funded by the UK Medical Research Council and the Wellcome Trust. This study makes use of data generated by the Wellcome Trust Case Control Consortium, funded by Wellcome Trust award 076113; a full list of the investigators who contributed to the generation of the data is available from www.wtccc.org.uk/. We thank the University of Virginia
}

for their support in genotyping samples from the Northern Irish GRID cohort, the IDDMGEN and T1DGEN cohorts from Finland and the Warren cohort using the T1DGC grant (U01 DK062418) and JDRF grant (JDRF 9-2011-530).

We thank N. J. Cooper for allowing us to use the imputation map generated to examine $\mathrm{AAD}$ variants across the entire genome.

Data availability The data analysed during the current study are available from the corresponding author on reasonable request.

Funding CW is funded by the Wellcome Trust (WT107881) and the Medical Research Council (MC_UP_1302/5). LB was supported by the Alan Turing Institute under the EPSRC grant EP/N510129/1.

Duality of interest The authors declare that there is no duality of interest associated with this manuscript.

Contribution statement JRJI carried out statistical analyses, wrote the manuscript and approved the final version. NMW helped with acquisition of the data and preparation of the data for analysis, revised the article critically and approved the final version of the manuscript. CW provided assistance implementing GUESSFM, input into the design of the study, provided code to carry out the haplotype analysis, and revised the article critically and approved the final version of the manuscript. LB provided statistical support throughout the project to JRJI and support with interpretation of data, revised the article critically and approved the final version. JAT directed research, provided biological expertise, helped with data acquisition and study design, revised the article critically and approved the final version of the manuscript. JAT is the guarantor of this work.

Open Access This article is distributed under the terms of the Creative Commons Attribution 4.0 International License (http:// creativecommons.org/licenses/by/4.0/), which permits unrestricted use, distribution, and reproduction in any medium, provided you give appropriate credit to the original author(s) and the source, provide a link to the Creative Commons license, and indicate if changes were made.

\section{References}

1. The Wellcome Trust Case Control Consortium (2007) Genomewide association study of 14,000 cases of seven common diseases and 3,000 shared controls. Nature 447:661-678

2. Todd JA, Walker NM, Cooper JD et al (2007) Robust associations of four new chromosome regions from genome-wide analyses of type 1 diabetes. Nat Genet 39:857-864

3. Barrett JC, Clayton D, Concannon P et al (2010) Genome-wide association study and meta-analysis finds over 40 loci affect risk of type 1 diabetes. Nat Genet 41:703-707

4. Onengut-Gumuscu S, Chen W-M, Burren O et al (2015) Fine mapping of type 1 diabetes susceptibility loci and evidence for colocalization of causal variants with lymphoid gene enhancers. Nat Genet 47:381-386

5. Fortune MD, Guo H, Burren O et al (2015) Statistical colocalization of genetic risk variants for related autoimmune diseases in the context of common controls. Nat Genet 47:839-846

6. Evangelou M, Smyth DJ, Fortune MD et al (2014) A method for gene-based pathway analysis using genomewide association study summary statistics reveals nine new type 1 diabetes associations. Genet Epidemiol 38:661-670 
7. Howson JMM, Cooper JD, Smyth DJ et al (2012) Evidence of gene-gene interaction and age-at-diagnosis effects in type 1 diabetes. Diabetes 61:3012-3017

8. Howson JMM, Rosinger S, Smyth DJ et al (2011) Genetic analysis of adult-onset autoimmune diabetes. Diabetes 60:2645-2653

9. Ziegler AG, Rewers M, Simell O et al (2013) Seroconversion to multiple islet autoantibodies and risk of progression to diabetes in children. JAMA 309:2473-2479

10. Qiao Q, Osterholm A-M, He B et al (2007) A genome-wide scan for type 1 diabetes susceptibility genes in nuclear families with multiple affected siblings in Finland. BMC Genet 8:84

11. Rich SS, Akolkar B, Concannon P et al (2009) Overview of the type I diabetes genetics consortium. Genes Immun 10:S1-S4

12. Bain SC, Todd JA, Barnett AH (1990) The British Diabetic Association-Warren Repository. Autoimmunity 7:83-85

13. Howie BN, Donnelly P, Marchini J (2009) A flexible and accurate genotype imputation method for the next generation of genomewide association studies. PLoS Genet 5(6):e1000529

14. Howie B, Marchini J, Stephens M (2011) Genotype imputation with thousands of genomes. G3 1:457-470

15. The 1000 Genomes Project Consortium (2015) A global reference for human genetic variation. Nature 526:68-74

16. Willer CJ, Li Y, Abecasis GR (2010) METAL: fast and efficient meta-analysis of genomewide association scans. Bioinformatics 26: 2190-2191

17. Conomos MP, Reiner AP, Weir BS, Thornton TA (2016) Modelfree estimation of recent genetic relatedness. Am J Hum Genet 98: $127-148$

18. Conomos MP, Thornton T (2016) GENESIS: GENetic EStimation and Inference in Structured samples (GENESIS): statistical methods for analyzing genetic data from samples with population structure and/or relatedness. R package, version 2.2.7

19. Svishcheva GR, Axenovich TI, Belonogova NM et al (2012) Rapid variance components-based method for whole-genome association analysis. Nat Genet 44:1166-1170

20. Pinheiro JC, Bates DM (2000) Mixed-effects models in S and SPLUS. Springer, Heidelberg https://doi.org/10.1007/b98882

21. Aulchenko YS, Ripke S, Isaacs A, van Duijn CM (2007) GenABEL: an R library for genome-wide association analysis. Bioinformatics 23:1294-1296

22. Banjamini Y, Hochberg Y (1995) Controlling the false discovery rate: a practical and powerful approach to multiple testing. J R Stat Soc B 57:289-300

23. Chen H, Wang C, Conomos MP et al (2016) Control for population structure and relatedness for binary traits in genetic association studies via logistic mixed models. Am J Hum Genet 98:653-666

24. Marchini J, Howie BN, Myers S et al (2007) A new multipoint method for genome-wide association studies by imputation of genotypes. Nat Genet 39:906-913

25. Wallace C, Cutler AJ, Pontikos N et al (2015) Dissection of a complex disease susceptibility region using a Bayesian stochastic search approach to fine mapping. PLoS Genet 11:e1005272

26. Bottolo L, Richardson S (2010) Evolutionary stochastic search for bayesian model exploration. Bayesian Anal 5:583-618

27. Bottolo L, Chadeau-Hyam M, Hastie DI et al (2013) GUESS-ing polygenic associations with multiple phenotypes using a GPUbased evolutionary stochastic search algorithm. PLoS Genet 9: 31003657
28. Yang J, Ferreira T, Morris AP et al (2012) Conditional and joint multiple-SNP analysis of GWAS summary statistics identifies additional variants influencing complex traits. Nat Genet 44:369-375

29. Stef van Buuren KG-O (2011) Mice: multivariate imputation by chained equations in R. J Stat Softw 45:1-67

30. Nejentsev S, Howson JM, Walker NM et al (2007) Localization of type 1 diabetes susceptibility to the MHC class I genes HLA-B and HLA-A. Nature 450:887-892

31. Nguyen C, Varney MD, Harrison LC, Morahan G (2013) Definition of high-risk type 1 diabetes HLA-DR and HLA-DQ types using only three single nucleotide polymorphisms. Diabetes 62:21352140

32. Miao F, Chen Z, Zhang L et al (2012) Profiles of epigenetic histone post-translational modifications at type 1 diabetes susceptible genes. J Biol Chem 287:16335-16345

33. Trynka G, Hunt KA, Bockett NA et al (2011) Dense genotyping identifies and localizes multiple common and rare variant association signals in celiac disease. Nat Genet 43:1193-1201

34. Jostins L, Ripke S, Weersma RK et al (2012) Host-microbe interactions have shaped the genetic architecture of inflammatory bowel disease. Nature 491:119-124

35. Sawcer S, Hellenthal G, Pirinen M et al (2011) Genetic risk and a primary role for cell-mediated immune mechanisms in multiple sclerosis. Nature 476:214-219

36. Morgan NG (2017) Bringing the human pancreas into focus: new paradigms for the understanding of type 1 diabetes. Diabet Med 34: 879-886

37. Johnson AL, Aravind L, Shulzhenko N et al (2009) Themis is a member of a new metazoan gene family and is required for the completion of thymocyte positive selection. Nat Immunol 10: 831-839

38. Asano A, Tsubomatsu K, Jung C-G et al (2007) A deletion mutation of the protein tyrosine phosphatase kappa (Ptprk) gene is responsible for T-helper immunodeficiency (thid) in the LEC rat. Mamm Genome 18:779-786

39. Iwata R, Sasaki N, Agui T (2010) Contiguous gene deletion of Ptprk and Themis causes T-helper immunodeficiency (thid) in the LEC rat. Biomed Res 31:83-87

40. Javierre BM, Burren OS, Wilder SP et al (2016) Lineage-specific genome architecture links enhancers and non-coding disease variants to target gene promoters. Cell 167:1369-1384

41. Beecham AH, Patsopoulos NA, Xifara DK et al (2013) Analysis of immune-related loci identifies 48 new susceptibility variants for multiple sclerosis. Nat Genet 45:1353-1360

42. Huang H, Fang M, Jostins L et al (2017) Fine-mapping inflammatory bowel disease loci to single-variant resolution. Nat Publ Gr 547:173-178

43. Fasano A (2005) Clinical presentation of celiac disease in the pediatric population. Gastroenterology 128:68-73

44. Cerutti F, Bruno G, Chiarelli F et al (2004) Younger age at onset and sex predict celiac disease in children and adolescents with type 1 diabetes: an Italian multicenter study. Diabetes Care 27:1294-1298

45. Bonifacio E, Mathieu C, Nepom GT et al (2016) Rebranding asymptomatic type 1 diabetes: the case for autoimmune beta cell disorder as a pathological and diagnostic entity. Diabetologia 60:6-9 